\title{
Behavioral Transition from Attack to Parenting in Male Mice: A Crucial Role of the Vomeronasal System
}

\author{
Kashiko S. Tachikawa, ${ }^{1,2}$ Yoshihiro Yoshihara, ${ }^{2}$ and Kumi O. Kuroda ${ }^{1}$ \\ ${ }^{1}$ Research Unit for Affiliative Social Behavior and ${ }^{2}$ Laboratory for Neurobiology of Synapse, RIKEN Brain Science Institute, Saitama 351-0198, \\ Japan
}

Sexually naive male mice show robust aggressive behavior toward pups. However, the proportion of male mice exhibiting pup-directed aggression declines after cohabitation with a pregnant female for 2 weeks after mating. Subsequently, on becoming fathers, they show parental behavior toward pups, similar to maternal behavior by mothers. To elucidate the neural mechanisms underlying this behavioral transition, we examined brain regions differentially activated in sexually naive males and fathers after exposure to pups, using c-Fos expression as a neuronal activation marker. We found that, after pup exposure, subsets of neurons along the vomeronasal neural pathway-including the vomeronasal sensory neurons, the accessory olfactory bulb, the posterior medial amygdala, the medioposterior division of the bed nucleus of stria terminalis, and the anterior hypothalamic area-were more strongly activated in sexually naive males than in fathers. Notably, c-Fos induction was not observed in the vomeronasal sensory neurons of fathers after pup exposure. Surgical ablation of the vomeronasal organ in sexually naive males resulted in the abrogation of pup-directed aggression and simultaneous induction of parental behavior. These results suggest that chemical cues evoking pup-directed aggression are received by the vomeronasal sensory neurons and activate the vomeronasal neural pathway in sexually naive male mice but not in fathers. Thus, the downregulation of pup pheromone-induced activation of the vomeronasal system might be important for the behavioral transition from attack to parenting in male mice.

\section{Introduction}

Parental care is crucial for the survival and proper development of young mammals. Mammalian neonates are born immature and require extensive care until weaning, such as nutrition, translocation, thermoregulation, and protection from hazards (Numan and Insel, 2003). Therefore, mothers are equipped with innate motivation for parental behavior. Even virgin female mice provide parental care, such as nest building and pup retrieving to the nest, to foster pups at the first encounter.

In contrast, behavioral responses toward pups of male mice differ depending on type of strain, experimental conditions, and reproductive context (Labov et al., 1985). In mouse strains such as CS-1, virgin males display aggression toward pups, sometimes leading to infanticide. However, after mating and cohabitation with pregnant females, CS-1 males begin to perform parental behaviors toward their offspring and even toward nonbiological

\footnotetext{
Received May 16, 2012; revised Jan. 28, 2013; accepted Feb. 5, 2013

Author contributions: K.S.T., Y.Y., and K.O.K. designed research; K.S.T. performed research; K.S.T. analyzed data; K.S.T., Y.Y., and K.O.K. wrote the paper.

This study was supported in part by Grants-in-Aid for Young Researchers (K.S.T., K.O.K.) and Innovative Areas (Systems Molecular Ethology) (Y.Y.) from the Ministry of Education, Culture, Sports, Science, and Technology of Japan. We thank Sayaka Komatsu, Sayaka Shindo, Ryuko Ohnishi, and Masayuki Nitta for technical assistance, Sachiko Mitsui, Rumiko Mizuguchi, and Tomomi Kaneko-Goto for experimental help and advice, Michael Numan, Yousuke Tsuneoka, Sachine Yoshida, Takefumi Kikusui, Alexandra V. Terashima, and Charles Yokoyama for helpful discussion, and the RIKEN Brain Science Institute Research Resource Center for maintenance of animals.

Correspondence should be addressed to Dr. Kashiko S. Tachikawa, Laboratory for Neurobiology of Synapse, RIKEN Brain Science Institute, 2-1 Hirosawa, Wako, Saitama 351-0198, Japan. E-mail: kashiko@brain.riken.jp.

DOI:10.1523/JNEUROSCI.2364-12.2013

Copyright $\odot 2013$ the authors $\quad 0270-6474 / 13 / 335120-07 \$ 15.00 / 0$
}

offspring (Kennedy and Elwood, 1988). Pup-directed aggression by males is thought to be an adaptive reproductive strategy that increases the males' inclusive fitness (vom Saal and Howard, 1982). Elimination of offspring terminates females' lactation and hastens ovulation, so that the chance of mating increases for males.

The behavioral transition from attack to parenting in male mice is a conspicuous biological phenomenon. Sensory stimuli from pups induce completely different behaviors in sexually naive males and fathers. In other words, social experience with females may leave a memory trace in males to influence the transition of their behavior toward pups. However, the precise mechanism underlying this transition remains essentially unknown.

The vomeronasal system mediates various social behaviors, such as copulation, male-male aggression, and pup avoidance in rodents (Fleming et al., 1979; Tirindelli et al., 2009). Although the involvement of the main olfactory system is also suggested (Brennan and Zufall, 2006), most pheromonal signals are detected in the vomeronasal organ (VNO) and transferred via the accessory olfactory bulb (AOB) to the medial amygdaloid nucleus (Me), posteromedial cortical amygdaloid nucleus, and bed nucleus of the stria terminalis (BST) (Gutiérrez-Castellanos et al., 2010). Eventually, pheromonal information reaches specific hypothalamic nuclei to elicit behavioral and physiological responses.

Here we looked for brain areas differentially activated by pup exposure between sexually naive males and fathers and found that the vomeronasal system plays a critical role in pup- 
directed aggression and the attack-to-parenting transition in male mice.

\section{Materials and Methods}

Animals. All experiments were approved by the Animal Care and Use Committee of RIKEN. C57BL/6 mice were bred and housed under a $12 \mathrm{~h}$ light/dark cycle in our animal facility. TEK-Fresh Standard bedding (Harlan) was used for animal housing. Purified paper chip (ALPHA-dri; Shepherd Specialty Papers) and cotton square material (Nestlets) were used for behavioral observation. Litters were weaned at $\sim 28 \mathrm{~d}$ of age, housed in same-sex groups, and used for experiments between 80 and $120 \mathrm{~d}$.

Pup retrieval assay. The pup retrieval assay was performed as described previously (Kuroda et al., 2007). Male responses toward pups were observed for $30 \mathrm{~min}$ and categorized into four types: (1) parenting, in which male mice retrieved all three pups to the nest and exhibited parental behavior continuously longer than $1 \mathrm{~min}$; (2) partial parenting, in which male mice retrieved one to three pups to the nest; (3) non-parenting, in which male mice ignored pups; and (4) attack, in which male mice attacked pups. When male mice displayed pup-directed aggression, observation was immediately terminated to rescue the pups. Pup-directed behavior was also evaluated with parental scores (5, parenting; 4 , threepup retrieval; 3 , two-pup retrieval; 2 , one-pup retrieval; 1 , non-parenting; and 0 , attack).

Five types of differently conditioned male mice were used: (1) sexually naive males, in which group-housed sexually naive males were isolated in a new home cage for $2 \mathrm{~d}$; (2-4) male mice experienced copulation and cohabitation with pregnant females for various time periods [ 7-10 d (2), 11-14 d (3), or 15-18 d (4)], were then isolated in a new home cage, and subjected to the pup retrieval assay $2 \mathrm{~d}$ after the birth of their pups; (5) father mice, in which males experienced copulation, cohabited with mates during gestation and delivery, spent $2 \mathrm{~d}$ with mates and pups, and were isolated in new home cages for $2 \mathrm{~d}$. Only sexually naive males that displayed pup-directed aggression within 5 min were used in experiments 2-5.

Pup exposure to male mice. Wire-mesh balls (tea balls, $45 \mathrm{~mm}$ diameter; Minex Metal) were used in pup exposure experiments to protect pups from males' attack. Approximately 60 holes $(3 \mathrm{~mm}$ diameter) were made on a ball so that male mice could contact and lick pups directly, without biting. Fathers that displayed parental behavior and sexually naive males that displayed pup-directed aggression were selected $2 \mathrm{~d}$ before the test and used for the subsequent c-Fos induction experiment. An empty wire-mesh ball was placed in the male's home cage $2 \mathrm{~d}$ before the assay and replaced with a ball containing three pups on the day of experiment. After $2 \mathrm{~h}$, the males were anesthetized, killed, and subjected to immunohistochemistry.

Immunohistochemistry. Immunohistochemistry was performed as described previously (Kimoto et al., 2005; Kuroda et al., 2007). Primary antibodies used were rabbit anti-c-Fos (1:20,000; Calbiochem) and rabbit anti-G $\alpha_{\mathrm{o}}$ (1:500 or 1:20,000; MBL). Biotin-conjugated goat antirabbit IgG (Vector Laboratories) and Alexa Fluor 488-conjugated goat anti-rabbit IgG (Invitrogen) were used as secondary antibodies. Images were captured with NanoZoomer 2.0-HT (Hamamatsu Photonics) and confocal microscope (FV1000; Olympus). Numbers of c-Fos-positive neurons were counted in every fifth of $20 \mu \mathrm{m}$ serial coronal sections of VNO bilaterally, every other of $40 \mu \mathrm{m}$ parasagittal sections of AOB unilaterally, and every third of $40 \mu \mathrm{m}$ coronal sections of other brain areas bilaterally using Neurolucida (MBF Bioscience) or Photoshop (Adobe Systems). Brain areas were determined according to the mouse brain atlas (Paxinos and Franklin, 2001).

Surgical ablation of VNO. Surgical ablation of VNO was performed by completely dissecting intact cartilaginous capsules enclosing VNO (Wysocki and Wysocki, 1995). The pup retrieval assay was performed 2 weeks after surgery. VNO ablation was confirmed by hematoxylin/eosin staining and lectin immunohistochemistry with horseradish peroxidaseconjugated soybean agglutinin (1:1000, J-Oil Mills).

Statistical analyses. We used SPSS Statistics 17.0 or Statcel3 (OMS) software. To compare pup-directed behaviors depending on social experiences, we applied Fisher's exact test to percentages of male mice exhib-
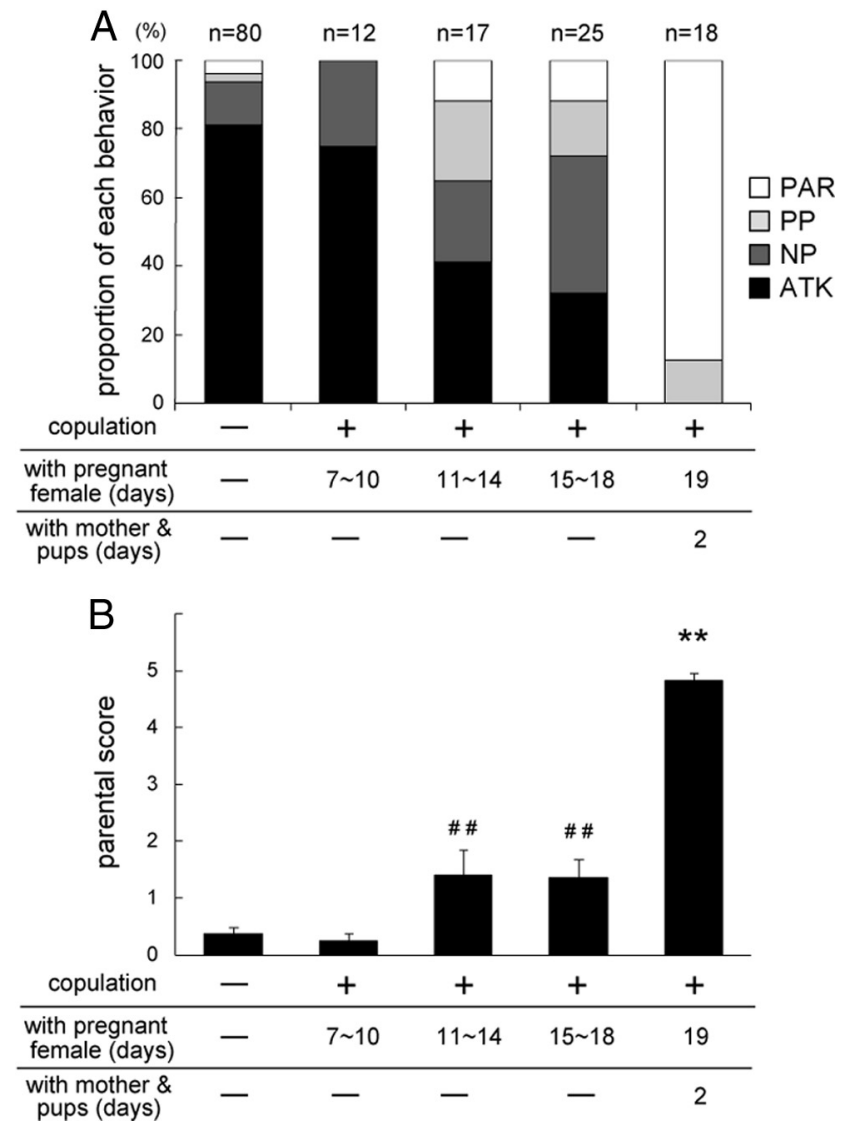

Figure 1. Experience-dependent behavioral transition from attack to parenting in C57BL/6 male mice. $A$, Percentages of male mice exhibiting each behavior toward pups. Males' responses toward pups were categorized into four types: (1) parenting (PAR, white); (2) partial parenting (PP, light gray); (3) non-parenting (NP, dark gray); and (4) attack (ATK, black). The number of subjects in each group is shown above each bar. $p<0.001$ (Fisher's exact test). $\boldsymbol{B}$, Parental scores of individual mouse groups. The data are represented as the mean \pm SEM. A Kuruskal-Wallis test revealed significant differences among groups $\left(H_{(4)}=71.71, p<0.001\right)$. ${ }^{\# \#} p<0.01$, different from sexually naive males; ${ }^{* *} p<0.01$, different from all other groups (Steel-Dwass test).

iting each behavior and a Kruskal-Wallis test, followed by a Steel-Dwass test as nonparametric post hoc analysis to the parental score. Numbers of c-Fos-positive cells were compared with Welch's ANOVA, followed by Welch's $t$ test. $p$ values of all multiple comparisons in the c-Fos experiment were adjusted appropriately using Holm's method. To evaluate the effect of VNO ablation on pup-directed behavior, Mann-Whitney's $U$ test was used.

\section{Results}

\section{Behavioral transition of C57BL/6 male mice from attack to parenting toward pups}

We first investigated pup-directed behavior of C57BL/6 male mice reared in our animal facility (Fig. 1). Pup-directed males' behavior was affected by social experience with females $(p<$ 0.001 ) (Fig. 1A). The majority $(81 \%)$ of sexually naive males displayed aggression toward pups. Seventy-five percent of males still exhibited pup-directed aggression after 7-10 d of postcopulatory cohabitation with pregnant mates, but this proportion declined after 11-14 d (41\%) and 15-18 d (32\%) (Fig. 1A, black columns). The parental score also significantly increased after 11-14 and 15-18 d of cohabitation compared with sexually naive males $(p<0.01)$ (Fig. $1 B)$. Thus, in C57BL/6 mice, the postcopulatory cohabitation for $11 \mathrm{~d}$ or longer with pregnant mates significantly suppressed pup-directed aggression. Subsequently, after 

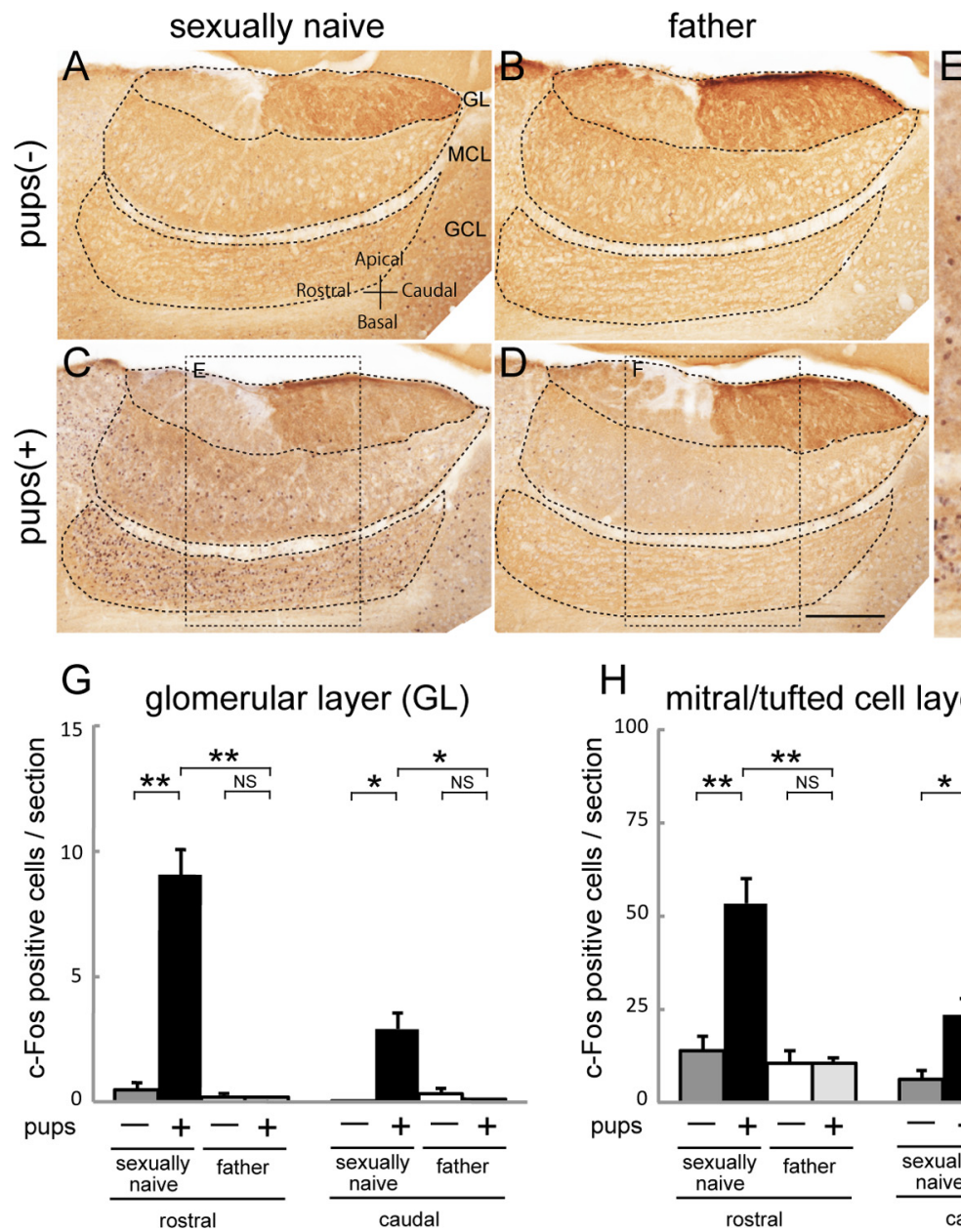

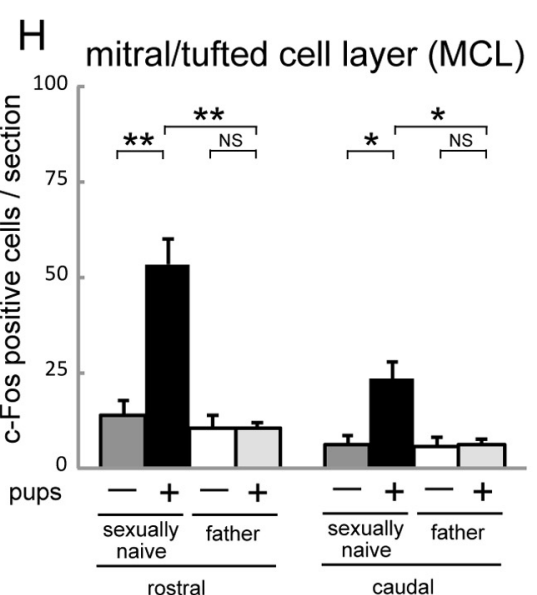

sexually naive

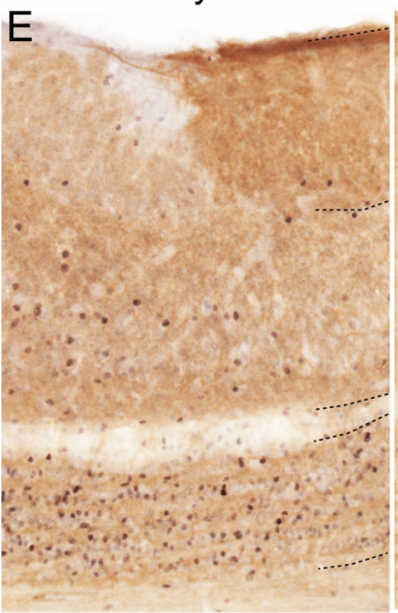

father

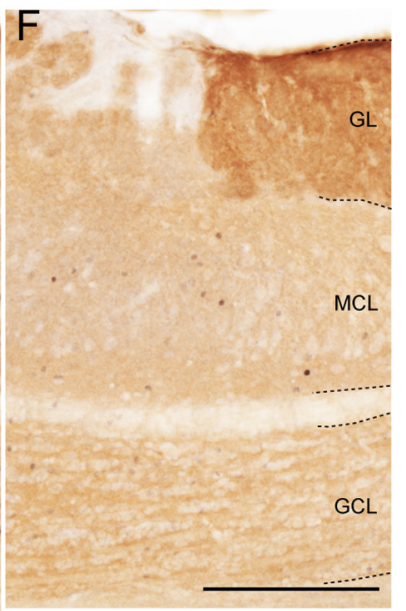

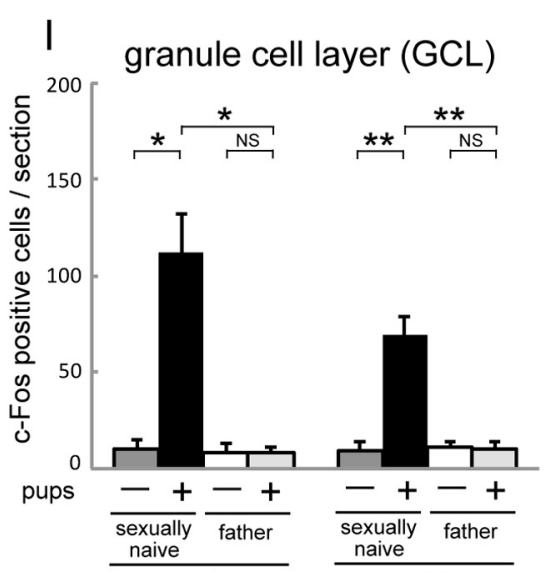

rostral

caudal

Figure 2. c-Fos expression in the AOB neurons of sexually naive males and fathers after pup exposure. $\boldsymbol{A}-\boldsymbol{F}$, Representative microscopic images of c-Fos (black) and $G \alpha_{0}$ (brown) expression in the $\mathrm{AOB}$ of sexually naive males $(\boldsymbol{A}, \boldsymbol{C})$ and fathers $(\boldsymbol{B}, \boldsymbol{D})$ in control condition $(\boldsymbol{A}, \boldsymbol{B})$ and after pup exposure $(\boldsymbol{C}, \boldsymbol{D})$. $\boldsymbol{E}, \boldsymbol{F}$, Higher magnification of $\boldsymbol{C}$ and $\boldsymbol{D}$. Scale bars, $100 \mu \mathrm{m}$. $\mathbf{G}-\boldsymbol{I}$, The numbers of c-Fos-positive cells in the glomerular layer $(G L)(G)$, mitral/tufted cell layer $(\mathrm{MCL})(\boldsymbol{H})$, and granule cell layer $(\mathrm{GCL})(\boldsymbol{I})$ in the rostral and caudal zones of the AOB in sexually naive males and fathers with or without pup exposure $(n=6)$. Welch's ANOVA revealed significant differences among groups in both rostral and caudal zones of the $\mathrm{GL}$ (rostral, $F_{(3,9.14)}=23.75, p<0.001 ;$ caudal, $\left.F_{(3,9.89)}=7.37, p=0.007\right)$, MCL (rostral, $F_{(3,9.61)}=12.40, p=0.001 ;$ caudal, $\left.F_{(3,10.58)}=4.58, p=0.027\right)$, and GCL (rostral, $F_{(3,10.09)}=7.59, p=0.006 ;$ caudal, $\left.F_{(3,10.69)}=10.68, p=0.002\right)$. Data are represented as mean $\pm S E M .{ }^{*} p<0.05,{ }^{* *} p<0.01$ (Welch's $t$ test followed by correction with Holm's method). NS, Not significant.

the males become fathers, pup-directed aggression was completely suppressed, and the majority (88\%) displayed parental behavior toward pups (Fig. 1A, white columns). The parental score also significantly increased in fathers compared with the other four groups $(p<0.01)$ (Fig. $1 B)$.

\section{Brain regions differentially activated in sexually naive males and fathers after pup exposure}

When a wire-mesh ball containing three pups was placed in fathers' home cages, they sniffed and licked it gently and started to build a nest. Four of 31 fathers (13\%) retrieved the pupcontaining ball to their nest. However, most sexually naive males were highly aroused, sniffed at higher frequencies, displayed eye squinting, and started to bite the pup-containing ball. Some sexually naive males displayed tail rattling as is observed in malemale aggression. After $2 \mathrm{~h}$ of pup exposure, male mice were killed and subjected to c-Fos immunohistochemistry. We compared various brain regions between sexually naive males and fathers with or without pup exposure and found differential c-Fos expression, particularly along the vomeronasal neural pathway.

We first noticed a clear difference in c-Fos induction between sexually naive males and fathers in the $\mathrm{AOB}$, the first relay station of the pheromonal information transfer in the brain. In sexually naive males, pup exposure resulted in a dramatic increase in the number of c-Fos-positive juxtaglomerular cells, mitral/tufted cells, and granule cells in both rostral and caudal zones of the AOB compared with control and fathers $\left({ }^{*} p<0.05,{ }^{* *} p<0.01\right)$ (Fig. 2A-I). In striking contrast, fathers showed no increase of c-Fos-positive cells after pup exposure (Fig. 2B, D,F-I).

Next, we examined c-Fos expression in secondary vomeronasal centers: the posterior region of the $\mathrm{Me}(\mathrm{MeP})$ and the medioposterior division of the BST (BSTMP). After pup exposure, a marked increase in the number of c-Fos-positive cells was observed in all subregions of the MeP and BSTMP of sexually naive males compared with control and fathers $\left({ }^{*} p<0.05,{ }^{* *} p<0.01\right)$ (Fig. $3 A-H, Q, R$ ). In fathers, pup exposure resulted in a smaller but significant increase of c-Fos-positive cells only in the dorsal subnucleus of the MeP (MePD) and the medial/intermediate subnuclei of the BSTMP (BSTMPM/I) $\left.{ }^{*} p<0.05,{ }^{*} p<0.01\right)$ (Fig. $3 A-H, Q, R$ ).

$\mathrm{MeP}$ and BSTMP neurons innervate specific hypothalamic structures, such as the medial preoptic area (MPA), medial preoptic nucleus (MPO), anterior hypothalamic area (AH), and ventromedial hypothalamic nucleus (VMH), leading to various 


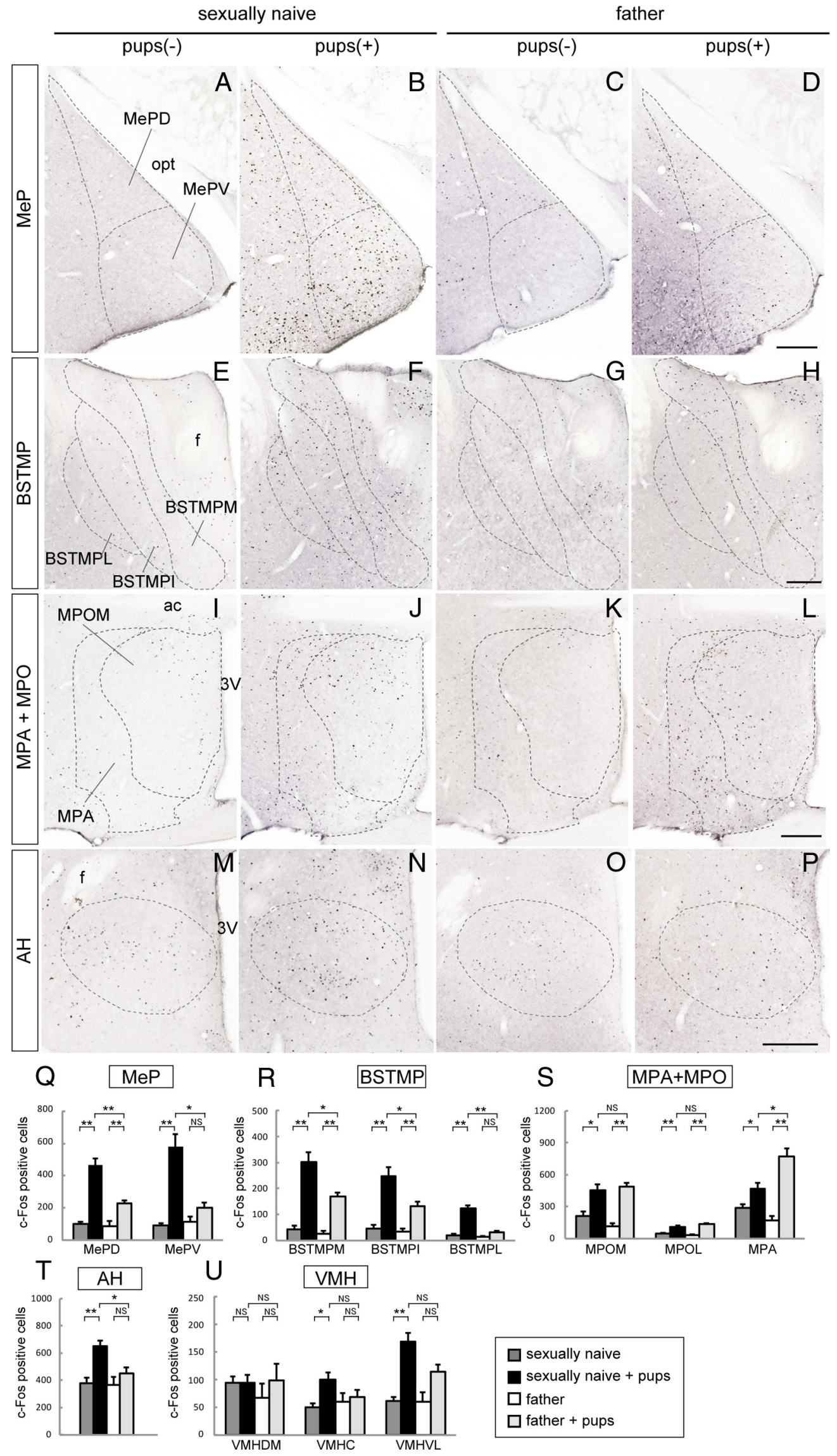

Figure 3. C-Fos expression in higher brain regions in sexually naive males and fathers after pup exposure. $\boldsymbol{A}-\boldsymbol{P}$, Representative microscopic images of c-Fos expression. $\boldsymbol{A}-\boldsymbol{D}$, The dorsal (MePD) and ventral (MePV) subregions of the MeP. opt, Optic tract. $\boldsymbol{E}-\boldsymbol{H}$, The medial (BSTMPM), intermediate (BSTMPI), and lateral (BSTMPL) parts of the (Figure legend continues.) 
behavioral and physiological responses, including copulation and aggression (Canteras et al., 1995; Dong and Swanson, 2004). In both sexually naive males and fathers, pup exposure resulted in a significant increase of c-Fos-positive cells in both the MPA and the MPO, which are collectively referred to the medial preoptic area $\left({ }^{*} p<0.05,{ }^{* *} p<0.01\right)$ (Fig. 3I-L,S). Notably, pup-exposed fathers contained a higher number of c-Fos-positive cells in the MPA compared with sexually naive males $\left({ }^{*} p<0.05\right)$ (Fig. 3I$L, S$ ). In the hypothalamus, pup exposure led to a significant increase in c-Fos-positive cells in the $\mathrm{AH}$ and the central/ ventrolateral subnucleus of the $\mathrm{VMH}$ of sexually naive males $\left({ }^{*} p<0.05,{ }^{* *} p<0.01\right)$ (Fig. $\left.3 M, N, T, U\right)$. In particular, the number of c-Fos-positive cells was significantly higher in the AH of sexually naive males than in fathers after pup exposure $\left({ }^{*} p<\right.$ 0.05 ) (Fig. $3 M-P, T$ ). There was no difference in the number of c-Fos-positive cells in the $\mathrm{AH}$ and the VMH between fathers and controls (Fig. 3O, P, T,U).

In conclusion, pup exposure resulted in activation of specific types of neurons along the vomeronasal pathway, including $\mathrm{AOB}, \mathrm{MeP}, \mathrm{BSTMP}$, and $\mathrm{AH}$, in sexually naive males compared with fathers.

\section{Comparison of c-Fos expression in the VNO}

Because a difference in c-Fos expression was detected at the level of the AOB, we next examined the activation of the vomeronasal sensory neurons (VSNs) after pup exposure. In sexually naive males, a higher number of c-Fos-positive VSNs compared with controls and fathers was observed $\left({ }^{* *} p<0.01\right)$ (Fig. $\left.4 A-I\right)$. c-Fos-expressing VSNs were observed in both the apical and basal zones of the VNO in pup-exposed sexually naive males (apical zone, $262 \pm 54$ cells; basal zone, $169 \pm 20$ cells; Welch's $t$ test, $p=$ 0.160 ). However, pup exposure induced little c-Fos expression in fathers' VSNs (Fig. 4D,I). These results suggest that putative pup pheromones activate a subpopulation of VSNs and induce the pup-directed aggression in sexually naive males but not in fathers.

\section{Effect of VNO ablation on pup-directed male behavior}

To confirm the importance of the VNO for pup-directed aggression, VNO-ablated male mice were subjected to the pup retrieval assay for 5 consecutive days. After the behavioral test, we histologically confirmed the complete ablation of VNO (Fig. 4J,K) and degeneration of vomeronasal axons (Fig. $4 L, M$ ). Parental scores significantly increased in $\mathrm{VNO}$-ablated sexually naive males compared with sham-operated sexually naive males throughout the $5 \mathrm{~d}$ assay $\left({ }^{* *} p<0.01\right)$ (Fig. $4 N$ ), indicating that pup-directed aggression was drastically suppressed by VNO ablation. Notably, 5 of 10 mice exhibited full parental behavior, and another four mice displayed partial parental behavior in the first assay. Eventually, all mice displayed full parental behavior except

\footnotetext{
$\leftarrow$

(Figure legend continued.) BSTMP. f, Fornix. I- $L$, MPA and the medial part of MPO (MPOM). ac, Anterior commissure; $3 V$, third ventricle. M-P, AH. Scale bars, $200 \mu \mathrm{m}$. Q-U, Quantification of c-Fospositive cells in each region $(n=6)$. Welch's ANOVA revealed significant differences among groups in the $\operatorname{MePD}\left(F_{(3,10.58)}=26.93, p<0.001\right), \operatorname{MePV}\left(F_{(3,10.07)}=13.07, p=0.01\right), \operatorname{BSTMPM}\left(F_{(3,10.79)}=\right.$ $29.06, p<0.001), \operatorname{BSTMPI}\left(F_{(3,10.75)}=16.19, p<0.001\right), \operatorname{BSTMPL}\left(F_{(3,10.78)}=20.53, p<0.001\right)$, $\operatorname{MPOM}\left(F_{(3,10.88)}=23.72, p<0.001\right), \operatorname{MPOL}\left(F_{(3,11.00)}=19.16, p<0.001\right), \operatorname{MPA}\left(F_{(3,10.79)}=16.93\right.$, $p<0.001), \mathrm{AH}\left(F_{(3,11.05)}=7.54, p=0.005\right), \operatorname{VMHC}\left(F_{(3,10.44)}=13.99, p=0.001\right)$, and VMHVL $\left(F_{(3,10.66)}=3.68, p=0.048\right)$ but not in VMHDM $\left(F_{(3,10.48)}=0.31, p=0.82\right)$. Data are represented as mean \pm SEM. ${ }^{*} p<0.05,{ }^{* *} p<0.01$ (Welch's t test followed by correction with Holm's method). MPOL, Lateral part of MPO; VMH, ventromedial hypothalamic nucleus; DM, dorsomedial; C, central; $\mathrm{VL}$, ventrolateral.
}

for one. Sham-operated sexually naive males continued to display pup-directed aggression (Fig. 4N), and VNO ablation did not affect the parental behavior of fathers (Fig. 4O). Thus, VNO ablation suppressed pup-directed aggression and simultaneously facilitated parental behavior in sexually naive males.

\section{Discussion}

Pup-directed aggression of C57BL/6 male mice was suppressed by postcopulatory cohabitation with pregnant females. The experience of being present during delivery and subsequent cohabitation with his mate and pups completely abrogated pupdirected aggression and instead induced parental behavior. This result is consistent with previous studies in outbred mice (Kennedy and Elwood, 1988) and rats (Brown, 1986) but not one study on C57BL/6 mice (Schneider et al., 2003). This difference might be attributable to differing experimental conditions, such as observation methods, places, and bedding (Kuroda et al., 2008).

We observed more intense c-Fos expression in central neurons along the vomeronasal pathway (AOB, MeP, and BSTMP) in sexually naive males compared with fathers (Figs. 2, 3). Hence, putative pup-derived pheromonal signals might be received more efficiently in sexually naive males. By analogy to the neural circuit mechanism of the pregnancy block phenomenon (Rosser and Keverne, 1985; Kaba et al., 1994), we originally postulated that social experience with a female and offspring may modulate pheromonal sensitivity of the $\mathrm{AOB}$ neurons in males, possibly through a plastic change in dendrodendritic synaptic transmission between mitral/tufted and granule cells. Unexpectedly, we found a difference in neuronal activation between sexually naive males and fathers in the VSNs, at the most peripheral level of the vomeronasal pathway (Fig. 4). It has been reported that sex steroids modulate pheromone-induced expression of immediateearly genes in the VNO (Halem et al., 2001). Thus, a pheromonesensing mechanism might be regulated at the level of the VSNs, depending on different social contexts and internal hormonal states. VNO ablation clearly suppressed pup-directed aggression and simultaneously induced parental behavior in sexually naive males (Fig. 4). Together with a previous report on the suppression of infanticide of male rats by VNO removal (Mennella and Moltz, 1988), our study suggests that abrogation of pup pheromone-induced activation of VSNs is necessary and sufficient for the attack-to-parenting transition in male mice. This result is reminiscent of a previous study describing that the vomeronasal nerve cut hastens the onset of maternal behavior in virgin female rats (Fleming et al., 1979). Thus, it is tempting to speculate that pup pheromones might activate the same neural pathways to suppress parental behaviors in both sexually naive male mice and female rats.

How are the memories of copulation and cohabitation with the pregnant mate stored and how is VSN activation suppressed in fathers? Several mechanisms are conceivable. VSNs of fathers may be less sensitive to pup-derived pheromones than those of sexually naive males. Downregulation of vomeronasal receptor expression and intracellular signaling or cell death of VSNs expressing receptors responsible for pup pheromones might occur in fathers' VNO through neural or hormonal mechanisms. Alternatively, uptake of pup pheromones into VNO lumen in fathers may be limited by a reduction of pumping controlled by vasomotor movement (Meredith and O'Connell, 1979). We also cannot rule out the possibility that father VSNs are excitable after pup exposure even without c-Fos induction. To elucidate the precise mechanism underlying social experience-dependent modulation 

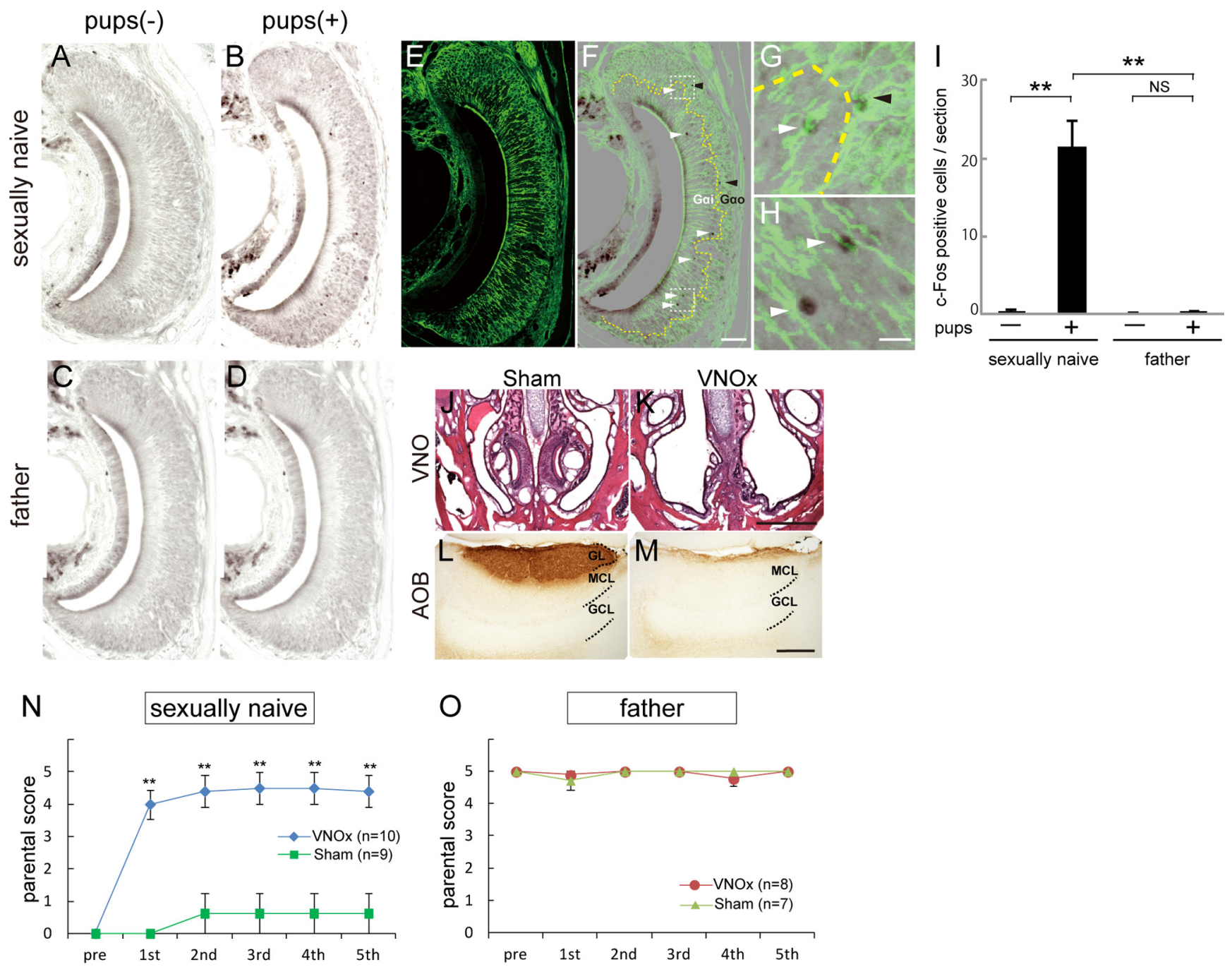

Figure 4. A crucial role of the VNO in the attack-to-parenting transition in male mice. $A-D, c-$ Fos expression (black) in VN0 of sexually naive males $(\boldsymbol{A}, \boldsymbol{B})$ and fathers $(\boldsymbol{C}, \boldsymbol{D})$ in control condition $(\boldsymbol{A}, \boldsymbol{C})$ and after pup exposure $(\boldsymbol{B}, \boldsymbol{D}) . \boldsymbol{E}-\boldsymbol{H}$, Double immunostaining of $\mathrm{VNO}$ section from sexually naive male mouse for $\mathrm{C}-\mathrm{Fos}$ and $\mathrm{G} \alpha_{0} . \boldsymbol{E}, \mathrm{G} \alpha_{0}$ expression (green) in the same section as $\boldsymbol{B} . \boldsymbol{F}$, Merged image of $\boldsymbol{B}$ and $\boldsymbol{E}$. White and black arrowheads denote c-Fos-positive/G $\alpha_{0}$-negative and c-Fos-positive/G $\alpha_{0}$-positive neurons, respectively. $\boldsymbol{G}, \boldsymbol{H}$, Enlarged views marked in $\boldsymbol{F}$. $\boldsymbol{I}$, Quantification of c-Fos-positive VSNs in sexually naive males and fathers with and without pup exposure $(n=6)$. Welch's ANOVA revealed a significant difference among groups $\left(F_{(3,9.24)}=12.84, p=0.001\right)$. Data are represented as mean $\pm S E M .{ }^{* *} p<0.01$ (Welch's $t$ test followed by correction with Holm's method). $J-M$, Surgical ablation of VN0. J, $K$, Coronal sections of the skulls from sham-operated ( $\boldsymbol{l}$ ) and VN0-ablated (VNOx) $(\boldsymbol{K})$ mice stained with hematoxylin and eosin. $\boldsymbol{L}, \boldsymbol{M}$, Parasagittal sections of the AOB from sham-operated $(\boldsymbol{L})$ and VN0-ablated $(\boldsymbol{M})$ mice labeled with horseradish peroxidase-conjugated soybean agglutinin (brown). $\boldsymbol{N}, \mathbf{O}$, Effect of VNO ablation (blue in $\boldsymbol{N}$, red in $\mathbf{0}$ ) on the pup-directed behaviors in sexually naive males $(\boldsymbol{N})$ and fathers $(\boldsymbol{O})$. The number of subjects is shown on the graph. Males' behaviors toward pups are plotted as parental scores. ${ }^{* *} p<0.01$ (Mann-Whitney's $U$ test). Scale bars: $\boldsymbol{F}, 50 \mu \mathrm{m} ; \boldsymbol{H}, 10 \mu \mathrm{m} ; \boldsymbol{M}, 200 \mu \mathrm{m}$.

of pheromone-induced VSN activation, other experimental strategies will be required, such as electro-vomeronasogram recording and calcium imaging.

The vomeronasal system plays critical roles in male-male aggression and maternal aggression (Tirindelli et al., 2009). The present study provides evidence that pup-directed aggression also depends on the vomeronasal system. Previous studies reported the involvement of the lateral septum, Me, BST, AH, and $\mathrm{VMH}$ in male-male and maternal aggressions (Nelson and Trainor, 2007). Most of these brain areas coincide with those activated by pup exposure in sexually naive males.

The MPA and MPO, which are known to be involved in parental behavior (Numan and Insel, 2003), were activated in both fathers and sexually naive males by pup exposure through a wiremesh ball. This finding is consistent with previous reports of direct pup exposure to male and female mice (Calamandrei and Keverne, 1994; Kuroda et al., 2007) and indirect pup exposure to paternal California mice (de Jong et al., 2009). Therefore, it can be concluded that MPA and MPO neurons are activated by pupderived sensory cues, regardless of gender or actual performance of parental behavior. Specifically, the MPA was the only area that showed higher activation in fathers than in sexually naive males after pup exposure. Thus, the strong activation of the MPA in fathers might reflect parental motivation. However, it is not clear whether the MPA and MPO neurons activated by pup exposure in sexually naive males represent the same neurons as those in fathers. The MPA and MPO subregions responsible for maternal behavior has been identified recently with detailed anatomical mapping (Tsuneoka et al., 2012). Additionally, we observed activation of the MePD and BSTMPM/I in the fathers after pup exposure, which was not reported in paternal California mice (de Jong et al., 2009), possibly because of differences in species, detection sensitivities, and experimental conditions.

VNO ablation led to not only suppression of pup-directed aggression but also expression of parental behavior in sexually naive males. Hence, we speculate that a pup might release two 
types of chemosensory cues: (1) aversive cues acting on the vomeronasal system and (2) attractive cues acting on the main olfactory system (Belluscio et al., 1998; Wang and Storm, 2011). In sexually naive males, aversive cues are received through the VNO and activate vomeronasal pathways for aggression, which dominate over the effect of attractive cues. However, in fathers and VNO-ablated males, the transmission of aversive cues is suppressed at the level of the VSNs and consequently the signals of attractive cues may become dominant, leading to the expression of parental behavior. Additional studies on this issue will pave the way to understanding the neural mechanisms for experiencedependent transitions of animal behavior in general.

\section{References}

Belluscio L, Gold GH, Nemes A, Axel R (1998) Mice deficient in G(olf) are anosmic. Neuron 20:69-81. CrossRef Medline

Brennan PA, Zufall F (2006) Pheromonal communication in vertebrates. Nature 444:308-315. CrossRef Medline

Brown RE (1986) Social and hormonal factors influencing infanticide and its suppression in adult male Long-Evans rats (Rattus norvegicus). J Comp Psychol 100:155-161. CrossRef Medline

Calamandrei G, Keverne EB (1994) Differential expression of Fos protein in the brain of female mice dependent on pup sensory cues and maternal experience. Behav Neurosci 108:113-120. CrossRef Medline

Canteras NS, Simerly RB, Swanson LW (1995) Organization of projections from the medial nucleus of the amygdala: a PHAL study in the rat. J Comp Neurol 360:213-245. CrossRef Medline

de Jong TR, Chauke M, Harris BN, Saltzman W (2009) From here to paternity: neural correlates of the onset of paternal behavior in California mice (Peromyscus californicus). Horm Behav 56:220-231. CrossRef Medline

Dong HW, Swanson LW (2004) Projections from bed nuclei of the stria terminalis, posterior division: implications for cerebral hemisphere regulation of defensive and reproductive behaviors. J Comp Neurol 471:396433. CrossRef Medline

Fleming A, Vaccarino F, Tambosso L, Chee P (1979) Vomeronasal and olfactory system modulation of maternal behavior in the rat. Science 203: 372-374. CrossRef Medline

Gutiérrez-Castellanos N, Martínez-Marcos A, Martínez-García F, Lanuza E (2010) Chemosensory function of the amygdala. Vitam Horm 83: 165-196. CrossRef Medline

Halem HA, Baum MJ, Cherry JA (2001) Sex difference and steroid modulation of pheromone-induced immediate early genes in the two zones of the mouse accessory olfactory system. J Neurosci 21:2474-2480. Medline

Kaba H, Hayashi Y, Higuchi T, Nakanishi S (1994) Induction of an olfactory memory by the activation of a metabotropic glutamate receptor. Science 265:262-264. CrossRef Medline

Kennedy HF, Elwood RW (1988) Strain differences in the inhibition of in- fanticide in male mice (Mus musculus). Behav Neural Biol 50:349-353. CrossRef Medline

Kimoto H, Haga S, Sato K, Touhara K (2005) Sex-specific peptides from exocrine glands stimulate mouse vomeronasal sensory neurons. Nature 437:898-901. CrossRef Medline

Kuroda KO, Meaney MJ, Uetani N, Fortin Y, Ponton A, Kato T (2007) ERKFosB signaling in dorsal MPOA neurons plays a major role in the initiation of parental behavior in mice. Mol Cell Neurosci 36:121-131. CrossRef Medline

Kuroda KO, Meaney MJ, Uetani N, Kato T (2008) Neurobehavioral basis of the impaired nurturing in mice lacking the immediate early gene FosB. Brain Res 1211:57-71. CrossRef Medline

Labov JB, Huck UW, Elwood RW, Brooks RJ (1985) Current problems in the study of infanticidal behavior of rodents. Q Rev Biol 60:1-20. CrossRef

Mennella JA, Moltz H (1988) Infanticide in the male rat: the role of the vomeronasal organ. Physiol Behav 42:303-306. CrossRef Medline

Meredith M, O'Connell RJ (1979) Efferent control of stimulus access to the hamster vomeronasal organ. J Physiol 286:301-316. Medline

Nelson RJ, Trainor BC (2007) Neural mechanisms of aggression. Nat Rev Neurosci 8:536-546. CrossRef Medline

Numan M, Insel TR (2003) The neurobiology of parental behavior. New York: Springer.

Paxinos G, Franklin K (2001) The mouse brain in stereotaxic coordinates. San Diego: Academic.

Rosser AE, Keverne EB (1985) The importance of central noradrenergic neurones in the formation of an olfactory memory in the prevention of pregnancy block. Neuroscience 15:1141-1147. CrossRef Medline

Schneider JS, Stone MK, Wynne-Edwards KE, Horton TH, Lydon J, O’Malley B, Levine JE (2003) Progesterone receptors mediate male aggression toward infants. Proc Natl Acad Sci USA 100:2951-2956. CrossRef Medline

Tirindelli R, Dibattista M, Pifferi S, Menini A (2009) From pheromones to behavior. Physiol Rev 89:921-956. CrossRef Medline

Tsuneoka Y, Maruyama T, Yoshida S, Nishimori K, Kato T, Numan M, Kuroda KO (2012) Functional, anatomical, and neurochemical differentiation of medial preoptic area subregions in relation to maternal behavior in the mouse. J Comp Neurol. Advance online publication. Retrieved February 13, 2013. doi:10.1002/cne.23251. CrossRef Medline

vom Saal FS, Howard LS (1982) The regulation of infanticide and parental behavior: implications for reproductive success in male mice. Science 215:1270-1272. CrossRef Medline

Wang Z, Storm DR (2011) Maternal behavior is impaired in female mice lacking type 3 adenylyl cyclase. Neuropsychopharmacology 36:772-781. CrossRef Medline

Wysocki CJ, Wysocki LM (1995) Surgical removal of the mammallian vomeronasal organ and its verification. In: Experimental cell biology of taste and olfaction (Spielman AI, Brand JG, eds), pp 49-57. Boca Raton, FL: CRC. 